\title{
Nonlinear Fourier transform for analysis of optical spectral combs
}

\author{
S. K. Turitsyn ${ }^{1,2}$, I. S. Chekhovskoy, ${ }^{1,3}$, and M. P. Fedoruk, ${ }^{1,3}$ \\ ${ }^{1}$ Novosibirsk State University, Novosibirsk 630090, Russia \\ ${ }^{2}$ Aston Institute of Photonic Technologies, Aston University, Birmingham B4 7ET, UK \\ ${ }^{3}$ Federal Research Center for Information and Computational Technologies, Novosibirsk 630090, Russia
}

(Dated: September 26, 2020)

\begin{abstract}
We characterise by the nonlinear Fourier transform (NFT) the build-up and dynamics of optical combs in the framework of the Lugiato-Lefever equation both for anomalous and normal dispersion. We demonstrate that NFT signal processing technique can simplify analysis of the formation of dissipative dark solitons and regimes exploiting modulation instability for a generation of coherent structures, by approximating comb with several discrete eigenvalues providing a platform for the analytical description of dissipative coherent structures.
\end{abstract}

Optical spectral comb technology enables a range of existing and emerging applications (see, [1] 7] and references therein). Due to their chip-scale size and potential for integration, micro-resonator frequency combs are attractive for generating of equally spaced, coherent (phase-locked) spectral lines, that is of particular interest for super-channel based optical communications. Multi-terabit per second coherent transmission has recently been demonstrated using both dissipative solitons and dark pulses in micro-resonators [8, 9]. The optical comb technology is based on the nonlinear science underlying building of coherent optical structures that ensure phase locking of spectral modes in the resonator.

Localized coherent structures and patterns formed from noise or unstable homogeneous states occur in a wide range of applications in physics and biology. Despite a great variety of these applications, they often have similar underlying mathematical models, that offer generic platform for new methods of analysis of localised temporal or spatial nonlinear waves. In this Letter, we demonstrate new applications of the nonlinear science method - inverse scattering transform (IST), also known as the nonlinear Fourier transform (NFT) to the characterisation of optical combs in several practically important configurations.

The master model governing the average evolution of the envelope of the optical field in nonlinear fibre- or micro-resonator (see for more details [17, 10, 11] and references therein) reads:

$$
i \frac{\partial \Psi}{\partial T}-\frac{\beta}{2} \frac{\partial^{2} \Psi}{\partial \tau^{2}}+|\Psi|^{2} \Psi=\left(-i+\zeta_{0}\right) \Psi+i f .
$$

Here $\Psi(T, \tau)$ is a slowly varying field amplitude, $T$ is a normalised time corresponding to the cavity round trips, $\tau$ is a dimensionless longitudinal coordinate related to the angular characteristic inside the micro-resonator (or the local time characteristic in case of fibre-resonator), $\zeta_{0}$ is the normalized laser detuning between the pump laser frequency and the cold-cavity resonance frequency, and $f$ is the normalised pump field amplitude; $\beta=\mp 1$ corresponds to, respectively, focusing/de-focusing cases (anomalous/normal dispersion in the context of fibre- optic applications). Eq. (1) is a mean-field model widely known as the Lugiato-Lefever equation (LLE) [12, was originally introduced in the context of plasma physics in [13], and first derived in the temporal domain in [14]. The left-hand-side of Eq. (1) (assuming that r.h.s is zero) presents the nonlinear Schrödinger equation (NLSE) that is integrable by IST [15, 16].

The IST/NFT method in application to NLSE is well documented and details can be found, for instance, in [15] 18, therefore, here we only briefly remind the key facts that will be used below. We limit IST/NFT consideration by fields $\Psi(T, \tau)$ decaying at $\tau \rightarrow \pm \infty$ for all $T$. The NLSE solutions $\Psi(T, \tau)$ are linked to the spectrum of a linear operator - the Zakharov-Shabat spectral problem (ZSSP) for potential $\Psi(T, \tau)$ and a spectral parameter $\lambda=\xi+i \eta$, as follows:

$$
\frac{\partial u}{\partial \tau}=-i \lambda+\Psi(T, \tau) v, \quad \frac{\partial v}{\partial \tau}=\beta \Psi^{*}(T, \tau) u+i \lambda v .
$$

For $\beta=-1$ the eigenvalue problem is non-Hermitian (ZSSP1) and for $\beta=1$ the operator is Hermitian (ZSSP2). Any solution of the $\operatorname{NLSE} \Psi(T, \tau)$ with $\beta=-1$ can be presented through the corresponding nonlinear spectrum of the ZSSP1, that in general, includes: (i) a continuous spectrum that is defined on the real axis of the complex plane $\lambda=\xi$ by the complex function $r(\xi)$, and (ii) a discrete spectrum that is described by $4 \times N$ real parameters (the set of complex-valued eigenvalues $\left\{\lambda_{n}\right\}$ having a positive imaginary part together with the complex-valued norming constants $\left.\left\{r_{n}\right\}\right)$. The discrete eigenvalues correspond to a soliton component containing of the field $\Psi(T, \tau)$, with $N$ being the total number of solitons. For the field $\Psi(T, \tau)$ that consists of a set of well separated solitons, each eigenvalue $\lambda_{n}$ specifies the soliton parameters: amplitude $2 \operatorname{Im}\left(\lambda_{n}\right)$, frequency $-2 \operatorname{Re}\left(\lambda_{n}\right)$, position $T_{n}=\log \left[\left|r_{n}\right| /\left(2 \operatorname{Im} \lambda_{n}\right)\right] /\left(2 \operatorname{Im} \lambda_{n}\right)$, and phase $\varphi_{n}=-\arg \left(i r_{n}\right)$.

For the case $\beta=-1$, the field energy can be presented as a sum of continuous (dispersive waves) and discrete 
(solitons) spectra of ZSSP1:

$$
\int_{-\infty}^{\infty}|\Psi(T, \tau)|^{2} d \tau=\sum_{n=1}^{N} 4 \eta_{n}+\frac{1}{\pi} \int_{-\infty}^{\infty} \log \left(1+|r(\xi)|^{2}\right) d \xi,
$$

where the left side of equality corresponds to the energy calculated in the temporal domain $E_{t}(T)$, while the right side includes a contribution of the discrete spectrum energy $E_{d}(T)$ and the continuous spectrum $E_{c}(T)$.

The initial idea (e.g. presented in [19 22]) behind using IST/NFT beyond the traditional integrable systems, was to exploit the fact that for some non-integrable (e.g. dissipative) models the Hamiltonian part of these equations is NLSE, and, thus, one can expect that the IST/NFT might still be a useful tool for analysis of the whole (nonHamiltonian) systems. The term NFT stresses the analogy with the traditional Fourier transform that is ubiquitous in science and engineering. Fourier transform has two key properties that make it such a widely used engineering method. First, use of the Fourier transform in a number of linear equations allows to solve them, e.g. by presenting a complex dynamics through a linear combination of non-interacting spectral components with simple evolution. Second, Fourier transform might be useful in simplifying the description of complex objects by presenting them via spectral harmonics. It was shown in [19, 21, 22, that in a similar manner IST/NFT can be employed not only for solving integrable equations, but also for the characterisation of localised coherent structures in dissipative systems in the anomalous dispersion regime. Note that in 23] periodic NFT was applied for analysis of static (output) optical comb profiles in the LLE model. However, in the case of periodic NFT localised structures have been presented by a large number of discrete eigenvalues that does not allow to reduce number of the effective degrees of freedom compared to conventional Fourier transform.

In this Letter, we demonstrate novel features and applications of NFT compared to the initial idea [19]21. We advance this emerging signal processing technique with the following novel applications of NFT to the characterisation of the dynamics of coherent structures during generating of optical combs: (i) for the first time we apply NFT based on ZSSP1 (with $\beta=-1$ in ZSSP (2)) to the analysis of dissipative dark solitons in Eq. (1) with normal dispersion. This is, evidently, a dramatic departure from the traditional IST, where the sign of $\beta$ must be the same for the NLSE and ZSSP used for its integration; (ii) we demonstrate how NFT can be used in the case of pulsed pumping waves; (iii) we characterise in terms of NFT spectrum generation of an optical comb through modulation instability-induced oscillations when detuning is switched to ensure a shift from unstable CW background to stable one; (iv) we demonstrate that steady-state dissipative dark soliton can be well approx- imated analytically by the N-soliton solutions of NLSE and a small number of parameters.

A typical solution of Eq. (1) includes a CW background $\Psi_{0}(T)$ and a solitonic part $\Psi_{1}(T, \tau)$. Evolution of the CW background $\Psi_{0}(T)$ is given by

$$
i \frac{\partial \Psi_{0}}{\partial T}+\left|\Psi_{0}\right|^{2} \Psi_{0}=\left(-i+\zeta_{0}\right) \Psi_{0}+i f .
$$

There are well-known solutions of 4 in the form of a constant CW background that can be found by solving Eq. (4) with $\frac{\partial \Psi_{0}}{\partial T}=0$, yielding algebraic equation on the stationary background $I_{0}=\left|\Psi_{0}\right|^{2}: \quad I_{0}^{3}-2 \zeta_{0} I_{0}^{2}+(1+$ $\left.\zeta_{0}^{2}\right) I_{0}-f^{2}=0$. There are three real roots, when the condition $f_{-}^{2} \leq f^{2} \leq f_{+}^{2}$ is satisfied, where

$$
f_{ \pm}^{2}=\frac{2}{27}\left(\zeta_{0}\left(\zeta_{0}^{2}+9\right) \pm \sqrt{\left(\zeta_{0}^{2}-3\right)^{3}}\right)
$$

and one real root otherwise. When $\zeta_{0}<\sqrt{3}$ only one real root exists 3 - 7 .

When localised structures that define comb have time scale much less than the round trip, it is possible to separate dynamics of the stable background field with nonzero boundary conditions (in $\tau$ ) from the evolution of the localised in time (vanishing boundary conditions) soliton content. This is possible when at large $|\tau|$ localised structures do not affect the $\mathrm{CW}$ background. Considering solution of the master model (1) as a sum of the uniform (in $\tau$ ) background $\Psi_{0}$ that depends only on $T$ and the soliton (localised in $\tau$ ) component $\Psi_{1}$, we can separate the evolution in $T$ of $\Psi_{0}$ governed by Eq. (4), and dynamics of the field $\Psi_{1}$ :

$$
i \frac{\partial \Psi_{1}}{\partial T}-\frac{\beta}{2} \frac{\partial^{2} \Psi_{1}}{\partial \tau^{2}}+\left|\Psi_{1}\right|^{2} \Psi_{1}=R\left[\Psi_{0}, \Psi_{1}\right],
$$

here the perturbative term $R$ describing deviations of Eq. (5) from the integrable NLSE has a form:

$R=\left(-i+\zeta_{0}\right) \Psi_{1}-2\left|\Psi_{0}\right|^{2} \Psi_{1}-2 \Psi_{0}\left|\Psi_{1}\right|^{2}-\Psi_{0}^{2} \Psi_{1}^{*}-\Psi_{0}^{*} \Psi_{1}^{2}$.

The proposed separation of the equations to the $\mathrm{CW}$ and solitonic parts only works when the background is stable. However, we will also show below how it can be used in the case of the unstable $\mathrm{CW}$ under the condition that the initial perturbation is localised in $\tau$ and detuning parameter is switched from unstable to stable background regimes before developing oscillations reach boundaries.

The Zakharov-Shabat problem (2) has been solved numerically by a hybrid method that includes computing discrete eigenvalues using phase jump tracking [24] and their subsequent refinement based on the Newton method with the exponential scheme 25].

Figure 1 depicts formation of a dissipative dark soliton in Eq. (1) with $\beta=1$ from the initial condition $\Psi(T=0, \tau)=2-1.8 \exp \left[-(\tau / 3.1)^{2}\right][26]$ in the case of the stable background $I_{0}=2$. Figure 1 shows that ZSSP1 

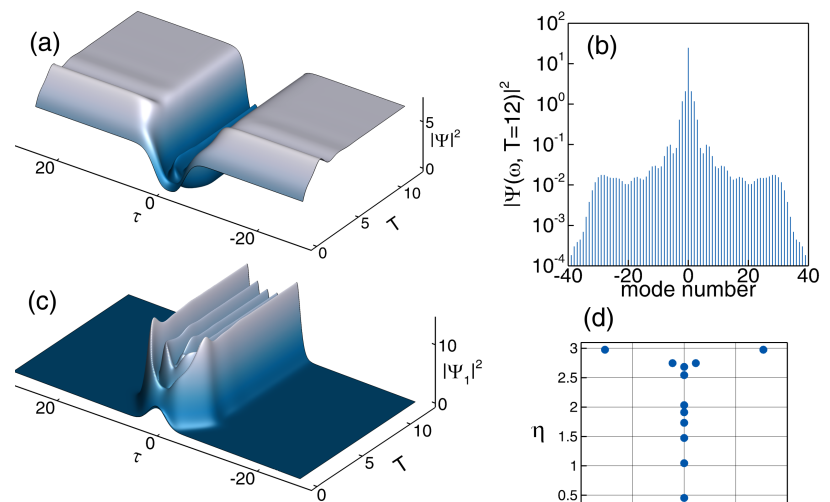

(d)
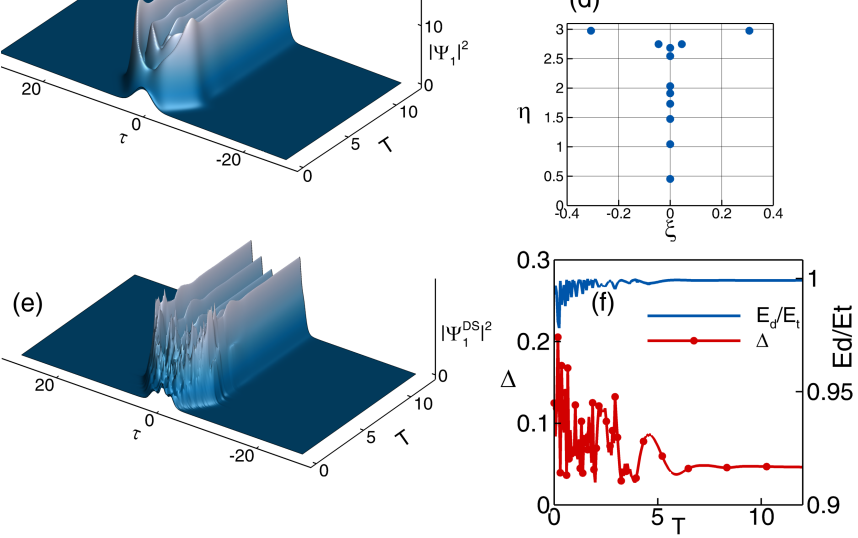

FIG. 1. Dissipative dark soliton on the stable CW background: (a) evolution with $T$ of the intensity $|\Psi(T, \tau)|^{2}$, (b) the spectral power density $|\Psi(T=12, \omega)|^{2}$, (c) intensity of the field without background $\left|\Psi_{1}(T, \tau)\right|^{2}$, (d) the nonlinear discrete spectrum at $T=12$, (e) the field reconstructed from the discrete spectrum only $\left|\Psi_{1}^{(D S)}(T=12, \tau)\right|^{2}$, (f) blue line - evolution with $T$ of the fraction of energy in the discrete spectrum $E_{d}(T) / E_{t}(T)$; relative integral $L_{2}$-norm of the difference between the field $\Psi_{1}$ and the field $\Psi_{1}^{(D S)}$ reconstructed from the nonlinear discrete spectrum (red line). Here $\Psi(T=0, \tau)=2-1.8 \exp \left(-(\tau / 3.1)^{2}\right), \beta=1, \zeta_{0}=6, f^{2}=8.5$.

(with $\beta=-1$ ) can be employed in the case of normal dispersion $(\beta=1)$ and that the dynamics of the field can be reconstructed with a reasonable accuracy from the discrete spectrum only. Figure 1(f) presents evolution with $T$ of a fraction of the energy $E_{d}(T) / E_{t}(T)$ containing in the discrete spectrum (blue line) and a relative error in terms of $L_{2}$-norm of the reconstruction of the total field using only discrete eigenvalues (red line). It is seen that dark soliton can be recovered with a good accuracy only from the discrete eigenvalues of the ZSSP1.

Next, we apply NFT characterisation to the generation of an optical comb through the modulation instability of the unstable CW background induced by localised oscillations. Figure 2 illustrates formation of optical comb when $\zeta_{0}=2$ (unstable CW) for $T<5$ and $\zeta_{0}=8.7666$ (stable CW) for $T \geq 5$. We consider the localised oscillating perturbations at $T=0 \Psi(0, \tau)=1.8 \exp \left(-2 \tau^{2}\right) \cos (0.3+5 \tau)$ that induce instability of the background. However, before oscillations reach the boundaries in the LLE model, the detuning $\zeta_{0}$ is switched to the stable $\mathrm{CW}$ background condition. As it is seen in Fig. 2, in the considered example, two discrete eigenvalues allow us to reconstruct the
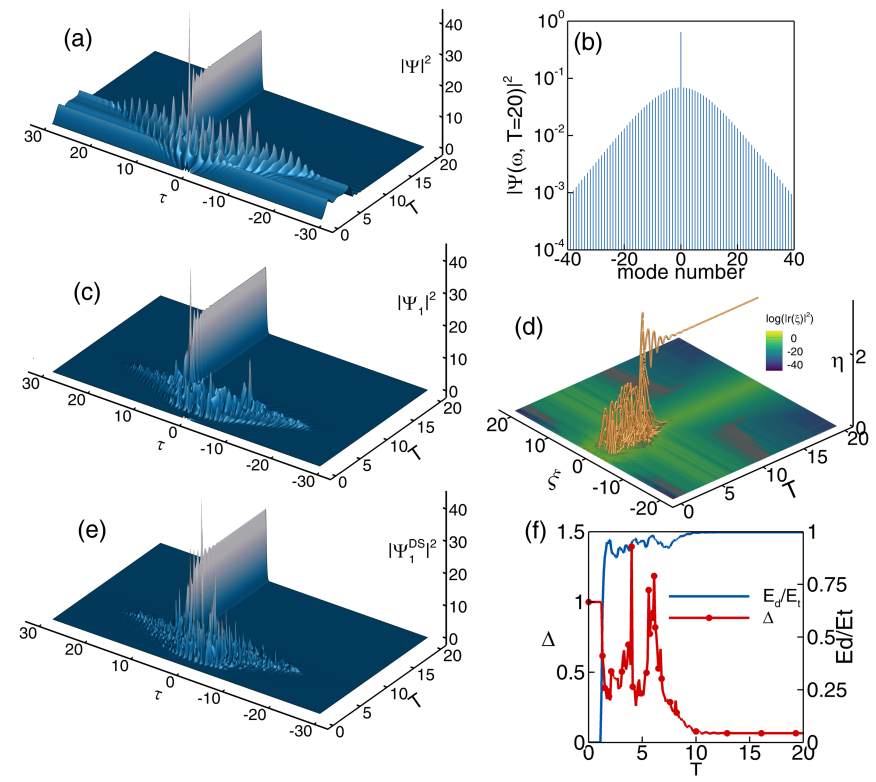

FIG. 2. Formation of comb through the modulation instability with switched detuning: (a) full field with background $|\Psi(T, \tau)|^{2}$, (b) comb spectral power density $|\Psi(T=12, \omega)|^{2}$, (c) $\left|\Psi_{1}(T, \tau)\right|^{2}$, (d) dynamics of the nonlinear spectrum with $T$ shown in the complex plane $\lambda=\xi+i \eta$ : the discrete spectrum (upper part) and the logarithm of $|r(\xi)|^{2}$ for continuous spectrum (counterplot), (e) the field reconstructed from the discrete spectrum only $\left|\Psi_{1}^{(D S)}(T=20, \tau)\right|^{2}$, (f) blue line evolution with $T$ of $E_{d}(T) / E_{t}(T)$; relative integral $L_{2}$-norm of the difference between the field $\Psi_{1}$ and the field $\Psi_{1}^{(D S)}$ reconstructed from the nonlinear discrete spectrum (red line). Here $\zeta_{0}=2$ for $T<5$ and $\zeta_{0}=8.7666$ for $T \geq 5$. Here $\beta=-1, f^{2}=3, \Psi(T=0, \tau)=1.8 \exp \left(-2 \tau^{2}\right) \cos (0.3+5 \tau)$.

total field with relatively good accuracy.

The proposed NFT analysis is well suited to optical comb generation 27] with pulsed pumping wave. In this case, there is no need to subtract $\mathrm{CW}$ background as the boundary conditions are decaying at large $|\tau|$. Consider the pumping wave in the form of a well-separated pulses of the form of $f(\tau)=1.9 \operatorname{sech}(\tau / 20)$, similar to example studied in [27. Figure 3 presents NFT characterisation of a comb formation from the initial Gaussian pulse $3 \exp \left(-\tau^{2} / 2\right)$. Steady-state field (Fig. $3(\mathrm{~b})$ ) has well-pronounced tails, leading to a discrete spectrum with one detached eigenvalue and a set of equally spaced eigenvalues with lower imaginary parts.

Finally, considering as an example a single dissipative dark soliton comb studied in [26] (shown in Fig. 4) we demonstrate that NFT approach can provide an analytical description for such stationary coherent structures in the non-integrable models. First, the optical field in the temporal domain was restored from the two discrete eigenvalues shown in Fig. 5(c) using the Darboux method. The analytical 2-soliton solution of NLSE corresponding to two discrete eigenvalues has the well-known 

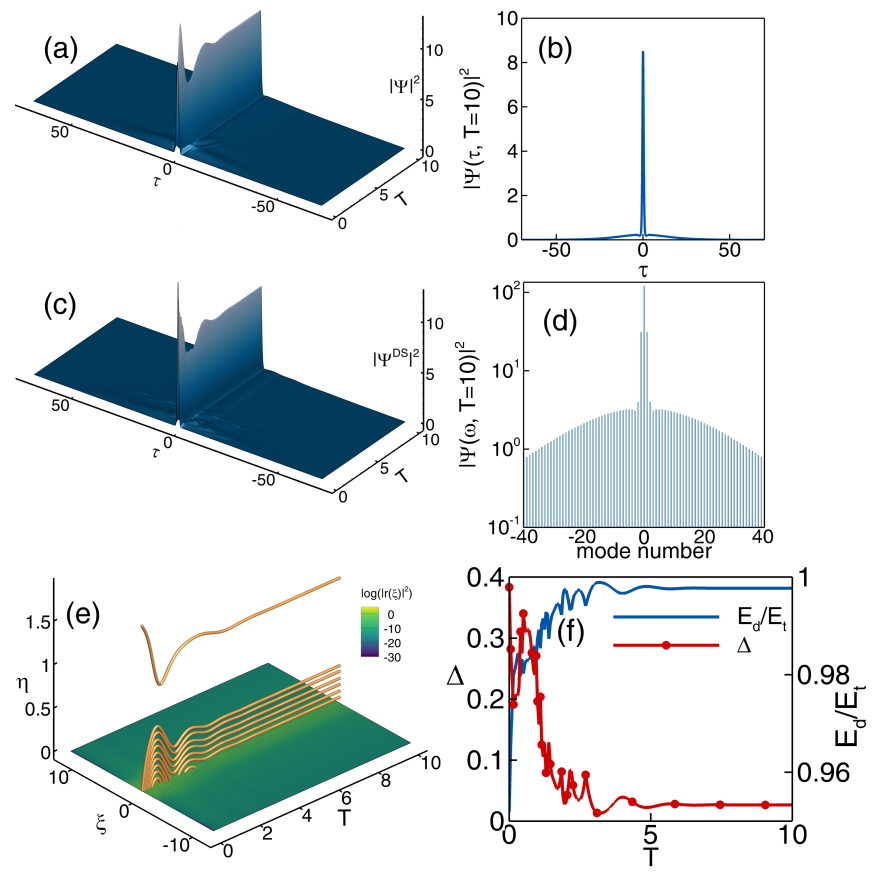

FIG. 3. Comb generation with a pulsed pumping wave $(f(\tau)=1.9 \operatorname{sech}(\tau / 20))$ generated from the Gaussian pulse $3 \exp \left(-\tau^{2} / 2\right)$ : (a) dynamics of intensity $|\Psi(T, \tau)|^{2}$, (b) formed comb shown at $T=10|\Psi(T=10, \tau)|^{2}$, (c) the field $\left|\Psi^{(D S)}(\tau, T)\right|^{2}$ reconstructed from the discrete spectrum shown in (e), (d) the spectral power density of comb $\mid \Psi(T=$ $12, \omega)\left.\right|^{2},(\mathrm{e})$ dynamics of the nonlinear spectrum shown as the evolution with $T$ in the complex plane $\lambda=\xi+i \eta$ : the discrete spectrum (upper part) and the logarithm of $|r(\xi)|^{2}$ for continuous spectrum (counterplot), (f) blue line - evolution with $T$ of $E_{d}(T) / E_{t}(T)$; relative integral $L_{2}$-norm of the difference between the field $\Psi$ and $\Psi^{(D S)}$ reconstructed only from the nonlinear discrete spectrum (red line). Here $\zeta_{0}=4, \beta=-1$.

exact form [17]:

$$
\Psi^{(2)}(\tau)=-2\left\langle\mathbf{A}(\tau)\left|\left[\mathbf{I}+\mathbf{M}(\tau)^{*} \mathbf{M}(\tau)\right]^{-1}\right| \mathbf{B}(\tau)\right\rangle .
$$

Here $\mathbf{I}$ is $2 \times 2$ identity matrix, $\mathbf{M}_{k, j}(\tau)=i r_{j} \frac{e^{-i\left(\lambda_{k}^{*}-\lambda_{j}\right) \tau}}{\lambda_{k}^{*}-\lambda_{j}}$ and 2-component vectors $\mathbf{A}(\tau)$ and $\mathbf{B}(\tau)$ are defined as: $\langle\mathbf{A}(\tau)|=\left\langle r_{1} e^{i \lambda_{1} \tau}, r_{2} e^{i \lambda_{1} \tau}\right|,\langle\mathbf{B}(\tau)|=\left\langle e^{i \lambda_{1} \tau}, e^{i \lambda_{1} \tau}\right|$. For the example considered in Fig. 5. $r_{1}=2.8661+7.4679 i$, $r_{2}=3.2519+2.3445 i, \lambda_{1}=1.4085 i, \lambda_{2}=0.6438 i$. Potential $\Psi^{(D S)}$ reconstructed from two eigenvalues provides analytical approximation of the dissipative dark soliton Fig. 4(a): $\Psi(T, \tau)=\Psi_{0}(T, \tau)+\Psi^{(D S)}(T, \tau)$.

Though a straightforward reconstruction of the potential $\Psi_{1}(T, \tau)$ from the discrete spectrum of ZSSP (2) allows approximation of the original field with good accuracy, it has disadvantages in the form of asymmetry (the original field is symmetric in $\tau$ ). It is well-known that the soliton modes are prone to the conversion of perturbations into jitter of discrete eigenvalue parameters. Therefore, reconstruction has to be enhanced by additional signal processing, discussion of which is beyond
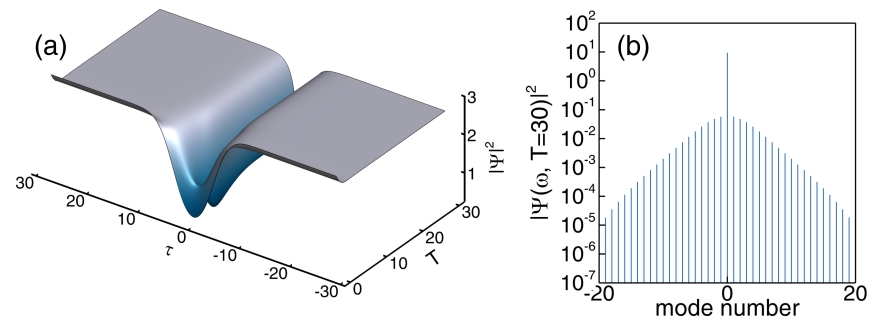

FIG. 4. Dissipative dark soliton: $\beta=1, \zeta_{0}=2.5, f^{2}=2.61$, $\Psi(T=0, \tau)=1.7-\exp \left(-(\tau / 4.4721)^{2}\right)$. (a) $|\Psi(\tau, T)|^{2}$, (b) the spectral power density $|\Psi(T=30, \omega)|^{2}$.
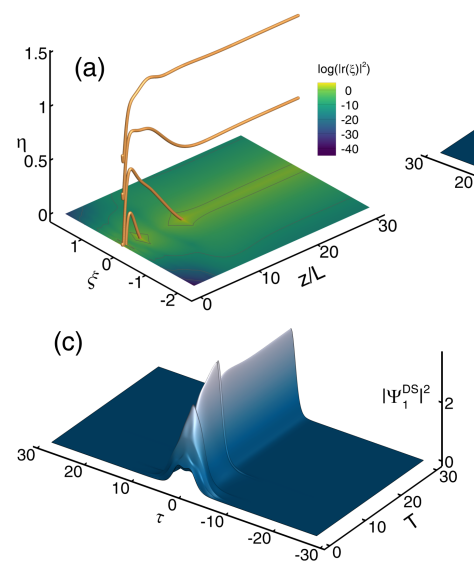

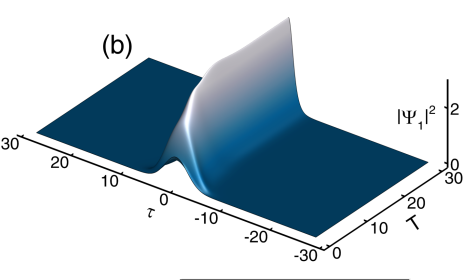

(d)

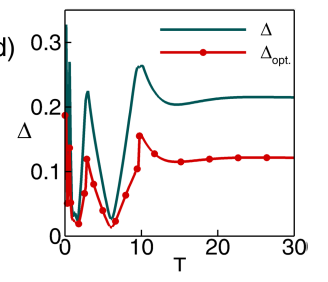

FIG. 5. Analytical approximation of dissipative dark soliton, same parameters as in Fig. 4. (a) dynamics in $T$ of the nonlinear spectrum in the complex plane $\lambda=\xi+i \eta$ : the discrete spectrum (upper part) and the logarithm of $|r(\xi)|^{2}$ for continuous spectrum (counterplot), (b) solution of Eq. (5) $\left|\Psi_{1}(T, \tau)\right|^{2}$, (c) the field $\left|\Psi_{1}^{(D S)}(T, \tau)\right|^{2}$ reconstructed from the discrete spectrum with using L2-optimization procedure, (d) relative integral $L_{2}$-norm of the difference between the field $\Psi_{1}$ and the field $\Psi_{1}^{(D S)}$ reconstructed from the nonlinear discrete spectrum by general procedure (purple line) and with using L2-optimization procedure (red line).

the scope of this Letter. Here, we apply the LevenbergMarquardt algorithm to minimize the $L_{2}$-norm of the deviation between original and reconstructed fields. This approach makes it possible to obtain symmetric field, and to halve the $L_{2}$-error (Fig. 5(d)). The optimal parameters read: $r_{1}=2.0767+5.198 i, r_{2}=1.9037+0.84997 i$, $\lambda_{1}=1.2797 i, \lambda_{2}=0.47663 i$ for $T=30$. We would like to stress that the analytical formula (6) well approximate here dissipative dark soliton in the non-integral system, as it is seen in Fig. 5. In this case, it is not necessary to solve the direct ZSSP to find the discrete spectrum, which, in the general case, is a complex resource-intensive task. However, the computed discrete spectrum provides a useful initial approximation for the optimization method. We also note that the number of discrete eigenvalues in this approach is not fixed and can be selected from the requirements of the reconstruction accuracy. 
In conclusion, we demonstrated that the NFT method based on the Zakharov-Shabat spectral problem used in the IST for NLSE with anomalous dispersion can be applied to the characterisation of optical combs in systems with both anomalous and normal dispersion, and with constant or pulsed pumping wave. We have shown that NFT technique can be used to analyse comb generation by the modulation instability of the plane wave when detuning parameter is switched from initially unstable background to the stable one. In the considered examples, NFT approach allowed us to present generated optical comb by several discrete eigenvalues. We demonstrated that NFT can provide the analytical description for some classes of dissipative dark solitons in situations when the most of the energy is contained in the discrete eigenvalues. Note an interesting link to the use of breather solution of NLSE for comb generation [28].

This work was supported by the Russian Science Foundation (grant No. 17-72-30006).

[1] K. Vahala, Nature 424, 839 (2003).

[2] T. J. Kippenberg, A. L. Gaeta, M. Lipson, and M. L. Gorodetsky, Science 361, eaan8083 (2018)

[3] L. Lugiato, F. Prati, M. L. Gorodetsky, and T. J. Kippenberg, Philosophical Transactions of the Royal Society A: Mathematical, Physical and Engineering Sciences 376 (2018).

[4] E. P. Grelu, Nonlinear Optical Cavity Dynamics: From Microresonators to Fiber Lasers (Wiley-VCH Verlag GmbH \& Co. KGaA, 2015).

[5] T. Hansson, D. Modotto, and S. Wabnitz, Phys. Rev. A 88, 023819 (2013).

[6] X. Xue, M. Qi, and A. M. Weiner, Nanophotonics 5, 244 (2016)

[7] T. Hansson and S. Wabnitz, Nanophotonics 5, 231 (2016)

[8] J. Pfeifle, V. Brasch, M. Lauermann, D. W. Yimin Yu, T. Herr, K. Hartinger, P. Schindler, J. Li, D. Hillerkuss, R. Schmogrow, C. Weimann, R. Holzwarth, W. Freude,
J. Leuthold, T. J. Kippenberg, and C. Koos, Nature Photonics 8, 375-380 (2014).

[9] Óskar B. Helgason, A. Fülöp, J. Schröder, P. A. Andrekson, A. M. Weiner, and V. Torres-Company, J. Opt. Soc. Am. B 36, 2013 (2019).

[10] S. Coen, H. G. Randle, T. Sylvestre, and M. Erkintalo, Opt. Lett. 38, 37 (2013)

[11] I. V. Barashenkov and Y. S. Smirnov, Physical Review E 54, 5707 (1996)

[12] L. A. Lugiato and R. Lefever, Physical Review Letters 58, 2209 (1987)

[13] K. Nozaki and N. Bekki, Physica 21D, 381 (1986).

[14] M. Haelterman, S. Trillo, and S. Wabnitz, Opt. Lett. 17, 745 (1992).

[15] V. E. Zakharov and A. B. Shabat, Journal of Experimental and Theoretical Physics 34, 62-69 (1972)

[16] M. J. Ablowitz, D. J. Kaup, A. C. Newell, and H. Segur, Studies in applied mathematics 53, 249 (1974).

[17] J. G. L. Lamb, Elements of soliton theory ((Wiley, 1980).

[18] M. J. Ablowitz and H. Segur, Solitons and the Inverse Scattering Transform (SIAM, 1981).

[19] I. S. Chekhovskoy, O. V. Shtyrina, M. P. Fedoruk, S. B. Medvedev, and S. K. Turitsyn, Physical Review Letters 122, 153901 (2019)

[20] P. Ryczkowski, M. Närhi, C. Billet, J.-M. Merolla, G. Genty, and J. M. Dudley, Nature Photonics 12, 221 (2018).

[21] S. Sugavanam, M. K. Kopae, J. Peng, J. E. Prilepsky, and S. K. Turitsyn, Nature Communications 10, 5663 (2019).

[22] S. K. Turitsyn, I. S. Chekhovskoy, and M. P. Fedoruk, Opt. Lett. 45, 3059 (2020).

[23] J. Wang, A.-G. Sheng, X. Huang, R.-Y. Li, and G.-Q. He, Chinese Physics B 29, 34207 (2020).

[24] I. Chekhovskoy, S. Medvedev, I. Vaseva, E. Sedov, and M. Fedoruk, "Introducing phase jump tracking - a fast method for eigenvalue evaluation of the direct ZakharovShabat problem," (2020).

[25] S. Medvedev, I. Vaseva, I. Chekhovskoy, and M. Fedoruk, Optics Express 28, 20 (2020)

[26] C. Godey, I. V. Balakireva, A. Coillet, and Y. K. Chembo, Physical Review A 89, 063814 (2014)

[27] M. Malinowski, A. Rao, P. Delfyett, and S. Fathpour, APL Photonics 2 (2017), 10.1063/1.4983113.

[28] B. Frisquet, A. Chabchoub, J. Fatome, C. Finot, B. Kibler, and G. Millot, Phys. Rev. A 89, 023821 (2014) 